\title{
Influência da Estrutura do Catalisador a base de Zirconoceno na Estereorregularidade e Propriedades do Polipropileno Formado
}

\author{
Mário R. Meneghetti, Madalena C. Forte e J airton Dupont
}

Resumo: Catalisadores de metalocenos com diferentes simetrias, cocatalisados por metilaluminoxanas (MAO), foram avaliados em diferentes temperaturas de polimerização do propileno. Os metalocenos com ponte interanelar, ou seja, o dicloreto de rac-etileno bis $\left(\eta^{5}\right.$-1-indenil) zircônio, com simetria $\mathrm{C}_{2}$, e o dicloreto de isopropilideno $\left(\eta^{5}\right.$-ciclopentadienil $)\left(\eta^{5}\right.$-9-fluorenil) zircônio, com simetria Cs, produziram, respectivamente, polipropileno isotático e sindiotático. O grau de taticidade desses polímeros diminui com o aumento da temperatura de polimerização. Os metalocenos sem ponte, dicloreto de bis $\left(\eta^{5}\right.$-ciclopentadienil)zircônio e bis $\left(\eta^{5}\right.$-indenil)zircônio, em qualquer uma das temperaturas investigadas, produziram, somente, polipropileno atático. A estereorregularidade dos polímeros foram determinadas por Ressonância Magnética Nuclear de ${ }^{13} \mathrm{C}$.

Palavras-chave: Metalocenos, zircônio, estereosseletividade, polipropileno, RMN.

\section{Introdução}

Desde a descoberta acidental do ferroceno em $1951^{1}$, uma vasta e variada gama de complexos de metais de transição, contendo o ligante $\eta^{5}$-ciclopentadienil e seus derivados, tem sido preparada. Inseridas nesta classe de compostos, várias famílias de metalocenos do grupo IV vêm sendo desenvolvidas nas duas últimas décadas. Os complexos de zircônio estereorrígidos, ou aqueles contendo ponte entre os ligantes ciclopentadienila, tem recebido especial atenção por parte da comunidade científica e industrial, em virtude, principalmente, das suas propriedades catalíticas frente a reação de polimerização estereosseletiva de $\alpha$-olefinas ${ }^{2}$, quando em presença de aluminoxanas.
A Figura 1 mostra a estrutura de alguns desses catalisadores com e sem ponte interanelar entre os ligantes.

Muitos estudos com estes catalisadores ZieglerNatta homogêneos, denominados de metalocenos, mostram que a estereoespecificidade do catalisador está intimamente relacionada com a estrutura do complexo organometálico ${ }^{3-8}$. Neste sentido, complexos catalíticos com diferentes simetrias devem produzir polímeros com estereoquímicas diferentes. Por exemplo, dos complexos metalocênicos mostrados na Figura 1, o complexo quiral rac$\mathrm{Et}(\mathrm{Ind})_{2} \mathrm{ZrCl}_{2}(1)$, com simetria $\mathrm{C}_{2}$ produz polímeros com estrutura isotática, o complexo metalocênico iPrCpFluZrCl 2 (2) com simetria Cs produz polímeros com estrutura sindiotática. 

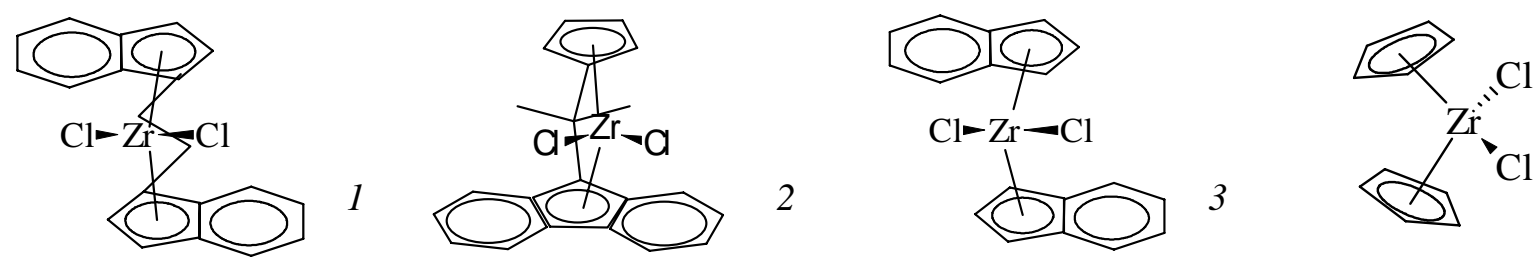

Figura 1. Estruturas dos catalisadores dicloreto de rac-etileno bis $\left(\eta^{5}-1\right.$-indenil)zircônio 1 ; dicloreto de isopropilideno $\left(\eta^{5}\right.$-ciclopentadienil) $\left(\eta^{5}-9\right.$ fluorenil)zircônio 2 ; dicloreto de bis( $\eta^{5}$-indenil)zircônio 3 e dicloreto de bis $\left(\eta^{5}\right.$-ciclopentadienil)zircônio 4 .

A estereoespecificidade observada nesses complexos estereorrígidos além de depender de aspectos geométricos da molécula, depende também de efeitos eletrônicos e estéricos ${ }^{9}$. Estes devem ser considerados, pois os mesmos podem levar a perda da estereoespecificidade do catalisador ou, ainda, levar a uma estereoespecificidade completamente diferente daquela considerando-se apenas a sua simetria. Por exemplo, o complexo dicloreto de rac-bis $\left(\eta^{5}-4\right.$ metilfluorenil)zircônio, apesar de não apresentar ponte interanelar ${ }^{10}$ produz polipropileno isotático com alto teor de cristalinidade. Isto se deve a existência de uma conformação com simetria $\mathrm{C}_{2}$, que apresenta rigidez, ou seja, há uma elevada energia envolvida na rotação da ligação "Metal-Cp".

Apesar do grande número de publicações na área de polimerização de $\alpha$-olefinas com catalisadores metalocênicos, nenhum estudo sistemático foi verificado por nós na literatura, até a realização do presente trabalho, relacionando a variação da estrutura do catalisador com a do polímero.

A análise de RMN de ${ }^{13} \mathrm{C}$ é tida como uma das ferramentas mais preciosas para o estudo das configurações estereoquímicas (microestrutura e taticidade) de polímeros, na elucidação do mecanismo de crescimento das cadeias poliméricas e, até mesmo, do peso molecular numérico médio $\left(\mathrm{M}_{\mathrm{n}}\right)$ do polímero $^{11-17}$.

Para uma análise da estereoquímica de um determinado polímero é necessário que as diferentes sequências de incorporação das olefinas estejam inequivocamente bem definidas no espectro de RMN de ${ }^{13} \mathrm{C}$ e, quanto maior for o comprimento dessas sequências, maiores serão as informações obtidas. Os carbonos do tipo $\mathrm{CH}_{3}, \mathrm{CH}_{2}$ e $\mathrm{CH}$ do polipropileno, em RMN de ${ }^{13} \mathrm{C}$, se localizam entre os deslocamentos 20 - 23, 46 - 49 e 28 - 30 ppm, respectivamente. Esse amplo deslocamento, em cada tipo de carbono, devese à vizinhança química dos mesmos, ou seja, a estereoquímica do polímero afeta significativamente e visivelmente os deslocamentos químicos dos carbonos que compõem a cadeia polimérica. Os diferentes deslocamentos, proporcionados pelos carbonos do tipo $\mathrm{CH}_{3}$, são os que mais sofrem influência em relação ao tipo de estereoquímica que o polímero apresenta, pelo menos em se tratando em termos de deslocamento químico. Assim, os diferentes tipos de carbono podem ser determinados e classificados dependendo da sua vizinhança.

Este trabalho teve como objetivo avaliar precursores catalíticos estereoespecíficos ou não na polimerização de polipropileno, e observar a influência da variação da temperatura sobre os mesmos. Para tanto quatro complexos de metalocenos à base de $\mathrm{Zr}$, com diferentes simetrias e graus de fluxionalidade (graus de esterorrigidez), foram sintetizados e empregados na polimerização de propeno.

\section{Experimental}

Os produtos químicos empregados como reagentes nas sínteses dos ligantes e complexos catalíticos foram adquiridos da Aldrich Chemical Co. e/ou Merck Co. com grau de pureza p.a. (para análise) ou p.s. (para síntese). O cocatalisador de metilaluminoxana $(5,5 \%$ de Al total em solução de tolueno) foi fornecido pela Witco Co. $n$-Hexano anidro foi fornecido pela OPP Petroquímica, e os solventes tetra-hidrofurano e éter etílico comerciais foram secos e destilados a temperatura de refluxo sob sódio metálico, utilizando-se como indicador benzofenona. O tolueno utilizado teve o mesmo tratamento que os últimos solventes, sendo primeiramente purificado para eliminação de tiofenos, conforme procedimento descrito na literatura ${ }^{18}$, e armazenado sob peneira molecular de $4 \AA$.

Todas as sínteses de ligantes e complexos catalíticos ${ }^{11,12,19-29}$ foram realizadas sob atmosfera de argônio, utilizando-se a técnica de Schlenk. A vidraria foi seca em estufa e resfriada sob fluxo de argônio. As soluções e os solventes foram manuseados e transferidos com seringas ou cânulas previamente 
purgadas com argônio. A agitação das soluções foi feita através de agitador magnético.

\section{Síntese de Ligantes e Catalisadores}

\section{1,2-bis(3-indenil)etileno - Et(Ind $)_{2}$}

Em um balão de três bocas de $250 \mathrm{~mL}$ foram adicionados na quantidade desejável indeno e $50 \mathrm{~mL}$ de THF. À temperatura de $0{ }^{\circ} \mathrm{C}$ foi adicionada gota a gota uma solução de butillítio 2,5 M em THF. À temperatura ambiente, foi adicionada gota a gota, sobre a suspensão do ânion orgânico a $-70{ }^{\circ} \mathrm{C}$, uma solução de 1,2-dibromoetano em $10 \mathrm{~mL}$ de THF. Deixou-se a mistura reacional sob agitação por toda a noite. À solução avermelhada foram adicionados $10 \mathrm{~mL}$ de $\mathrm{NH}_{4} \mathrm{Cl}_{(\mathrm{aq})}$ e $10 \mathrm{~mL}$ de éter de petróleo. A fase orgânica foi lavada com 3 alíquotas de $10 \mathrm{~mL}$ de salmoura e seca em $\mathrm{MgSO}_{4}$. Em seguida, a solução foi levada ao vácuo, havendo a formação de um precipitado bege, que foi recristalizado em acetona/etanol a $-5{ }^{\circ} \mathrm{C}$. O rendimento da reação foi de $65 \%$.

$\mathrm{RMN}^{1} \mathrm{H}\left(\mathrm{CDCl}_{3}\right): 7,52-7,18$ (m, 8H, arom.); 6,30 (s, 2H, olef.); 3,36 (s, 4H, $\mathrm{CH}_{2}$ ciclo); 2,95 (s, 4H, $\mathrm{CH}_{2}$ ponte).

\section{Dicloreto de rac-etilenobis( $\eta^{5}$-1-indenil)zircônio - Et(Ind $)_{2} \mathrm{ZrCl}_{2}$}

A uma solução de 1,2-bis(indenil)etileno em THF, a $-78{ }^{\circ} \mathrm{C}$, foi adicionada gota a gota uma solução de butillítio 2,3 M em THF. A mistura reacional foi aquecida à temperatura ambiente por uma hora, passando a ter coloração castanha. Paralelamente, foi preparada uma solução do aduto $\mathrm{ZrCl}_{4}$.2THF em 50 $\mathrm{ml}$ de tetrahidrofurano ${ }^{30}$. Estas duas soluções foram adicionadas gota a gota (1 gota/seg), simultaneamente, sobre $25 \mathrm{~mL}$ de THF. Após, $\mathrm{HCl}_{(\mathrm{g})}$ foi borbulhado por 3 min na solução e os voláteis foram retirados a vácuo. $\mathrm{O}$ precipitado amarelo obtido foi separado com o auxílio de éter etílico e filtrado em funil de vidro sinterizado. Foi lavado com $5 \mathrm{~mL}$ de $\mathrm{HCl} 4 \mathrm{~N}, 5 \mathrm{~mL}$ de água e, finalmente, com 2 alíquotas de $5 \mathrm{~mL}$ de etanol. O rendimento da reação foi de aproximadamente $20 \%$.

$\mathrm{RMN}^{1} \mathrm{H}\left(\mathrm{CDCl}_{3}\right): 7,70-7,18$ (m, 8H, arom.); 6,61 e 6,23 (2d, 4H, Cp); 3,87 (s, 4H, ponte).

\section{Dicloreto de bis $\left(\eta^{5} \text {-indenil)zircônio - (Ind }\right)_{2} \mathrm{ZrCl}_{2}$}

A síntese e tratamento deste complexo são bastante semelhantes aos realizados para o complexo
$\mathrm{Et}(\mathrm{Ind})_{2} \mathrm{ZrCl}_{2}$. Entretanto, não houve a necessidade de se adicionar, ao mesmo tempo, a um terceiro frasco, as duas soluções reagentes (o sal orgânico de Li e o cloreto metálico). O cuidado em se adicionar simultaneamente as duas soluções é importantíssimo para o caso do catalisador com ponte, devido ao fato do ligante com ponte, na forma de ânion, apresentar dois sítios de coordenação que podem reagir e coordenar-se a um mesmo metal de transição. Assim, a uma solução de indeno em THF, a $0{ }^{\circ} \mathrm{C}$, foi adicionada, gota a gota, uma solução de butillítio 1,6 M. A mistura reacional foi aquecida à temperatura ambiente. Paralelamente foi preparada uma solução do aduto $\mathrm{ZrCl}_{4}$. $2 \mathrm{THF}$ em $25 \mathrm{~mL}$ de tetrahidrofurano. A solução do ânion orgânico foi adicionada gota a gota, a $0{ }^{\circ} \mathrm{C}$, sobre a solução do aduto. Após, $\mathrm{HCl}_{(\mathrm{g})}$ foi borbulhado por 3 min e os voláteis foram retirados a vácuo. O precipitado amarelo obtido foi separado com o auxílio de éter etílico e filtrado em funil de vidro sinterizado. Foi lavado com $25 \mathrm{~mL}$ de $\mathrm{HCl} 4 \mathrm{~N}, 25$ $\mathrm{mL}$ de água e, finalmente, com 2 alíquotas de $25 \mathrm{~mL}$ de etanol. O rendimento foi de aproximada mente $40 \%$.

$\mathrm{RMN}{ }^{1} \mathrm{H}\left(\mathrm{CDCl}_{3}\right): 7,60$ e 7,25 (2m, 8H, arom.); 6,45 e 6,10 ( $\mathrm{t}$ e d, $2 \mathrm{H}$ e $4 \mathrm{H}, \mathrm{Cp}$ ).

\section{2-(3-ciclopentadienil)-2-(9-fluorenil)propano e 2- (2-ciclopentadienil)-2-(9-fluorenil) isopropilideno - iPrCpFlu}

Em um balão Schlenk de $250 \mathrm{~mL}$ foram adicionados fluoreno em quantidade desejável e $80 \mathrm{~mL}$ de THF seco. Sobre esta solução, foi adicionada uma solução de butillítio $1,4 \mathrm{M}$ em THF, gota a gota, a $0{ }^{\circ} \mathrm{C}$. A mistura foi deixada em agitação por $30 \mathrm{~min}$ a temperatura ambiente. Em outro balão, foi preparada uma solução de dimetilfulveno em THF, que foi adicionada à solução anterior, gota a gota, com o auxílio de cânula. A mistura reacional foi deixada em agitação durante a noite. A hidrólise dessa solução foi feita com $50 \mathrm{~mL}$ de $\mathrm{NH}_{4} \mathrm{Cl}_{(\mathrm{aq})}$ e a fase orgânica foi lavada com 3 alíquotas de $20 \mathrm{~mL}$ de água. Os voláteis foram retirados em rota vapor e o ligante foi cristalizado a $-35{ }^{\circ} \mathrm{C}$ em presença de éter etílico. O rendimento da reação foi de $60 \%$.

$\mathrm{O}$ espectro de $\mathrm{RMN}{ }^{1} \mathrm{H}$ do produto obtido assinala a presença dos dois isômeros acima intitulados, em uma razão de 1,7:1. Essa mistura não inviabiliza a reação seguinte, nem necessita de purificação, ao reagirse os dois isômeros com BuLi, aromatizando os anéis Cp, é produzido o mesmo composto, ou seja, o mesmo diânion orgânico. 
RMN ${ }^{1} \mathrm{H}\left(\mathrm{CDCl}_{3}\right)$ do isômero A: 7,69-7,00 (1d$2 \mathrm{~m}, 8 \mathrm{H}$, arom); 6,87, 6,57 e 5,83 (dd, dd e t, $3 \mathrm{H}$, olef.); 4,11 (s, 1H, CH); 3,00 (d, 2H, $\left.\mathrm{CH}_{2}\right) ; 0,99$ (s, $\left.6 \mathrm{H}, \mathrm{CH}_{3}\right)$.

$\mathrm{RMN}{ }^{1} \mathrm{H}\left(\mathrm{CDCl}_{3}\right)$ do isômero B: 7,69-7,00 (1d$2 \mathrm{~m}, 8 \mathrm{H}$, arom); $6,45,6,37$ e $6,05(\mathrm{dd}, \mathrm{dd}$ e $\mathrm{t}, 3 \mathrm{H}$, olef.); 4,05 (s, 1H, CH); 3,10 (d, 2H, $\left.\mathrm{CH}_{2}\right) ; 1,02$ (s, $\left.6 \mathrm{H}, \mathrm{CH}_{3}\right)$.

\section{Dicloreto de isopropilideno $\left(\eta^{5}\right.$-ciclopentadienil) $\left(\eta^{5}\right.$-9-fluorenil)zircônio - iPrCpFluZrCl$l_{2}$}

Em um balão Schlenk de $250 \mathrm{~mL}$ foram adicionados o ligante iPrCpFlu em quantidade desejável e $100 \mathrm{~mL}$ de THF seco. A esta solução foi adicionado, gota a gota, a $0{ }^{\circ} \mathrm{C}$, uma solução de butillítio $1,2 \mathrm{M}$ em THF. A mistura reacional foi aquecida à temperatura ambiente e mantida sob agitação até que não houvesse mais evolução de gás. Os voláteis foram retirados a vácuo em banho-maria $\left(\approx 50^{\circ} \mathrm{C}\right)$, obtendose um precipitado vermelho sangue. Neste momento não tem-se mais a mistura de isômeros, mas sim um único produto, o diânion orgânico iPrCpFluLi 2 . O precipitado foi lavado com 2 alíquotas de $40 \mathrm{~mL}$ de hexano e novamente seco a vácuo. Sobre o sólido vermelho foram adicionados $100 \mathrm{~mL}$ de $\mathrm{CH}_{2} \mathrm{Cl}_{2}$, e após a homogeneização da suspensão vermelha, foi adicionado tetracloreto de zircônio a $-78^{\circ} \mathrm{C}$. A mistura reacional foi aquecida à temperatura ambiente. Após, a suspensão foi filtrada em funil de vidro sinterizado tipo Schlenk. O filtrado foi mantido a $-20^{\circ} \mathrm{C}$, obtendose cristais vermelhos. Este precipitado foi separado da solução com o auxílio de cânula, seco a vácuo e identificado por $\mathrm{RMN}$ de ${ }^{1} \mathrm{H}$. O rendimento foi de $15 \%$.

RMN ${ }^{1} \mathrm{H}\left(\mathrm{CD}_{2} \mathrm{Cl}_{2}\right): 8,10-7,15$ (2d, 2t, $8 \mathrm{H}$, arom); 6,23 e 5,69 (2t, 4H, Cp); 2,30 (s, 6H, $\left.\mathrm{CH}_{3}\right)$.

\section{Polimerizações}

As polimerizações de propeno foram realizadas em reator de vidro encamisado de $0,5 \mathrm{~L}$ equipado com agitador mecânico, utilizando-se como gás inerte nitrogênio. O reator antes de cada reação de polimerização era tratado com isoparafina (ISOPAR), à $60{ }^{\circ} \mathrm{C}$ sob vácuo, para eliminação de umidade $\mathrm{e}$ após era purgado com nitrogênio. A temperatura de reação foi controlada por banho termostatizado e a adição dos componentes catalíticos foi feita em atmosfera de propeno. A ordem de adição dos componentes no reator foi tolueno, cocatalisador e catalisador em solução de tolueno. As quantidades relativas a cada componente estão especificadas na Tabela 1, nos resultados e discussão.

\section{Caracterização dos polímeros obtidos}

As análises de Ressonância Magnética Nuclear de Carbono-13 (RMN ${ }^{13} \mathrm{C}$ ) dos polímeros foram realizadas em um espectrômetro VARIAN VXR 200, sendo os deslocamentos químicos $(\delta)$ expressos em partes por milhão (ppm) e calculados em relação ao pico do tetra-metilsilano (TMS, $\delta=0 \mathrm{ppm}$ ). Os parâmetros do espectrômetro foram freqüência de $50.309 \mathrm{MHz}$, tempo de aquisição de $1,4 \mathrm{~s}$ e de relaxação de $3 \mathrm{~s}$, ângulo de pulso de $68^{\circ}$ e uma temperatura de $90{ }^{\circ} \mathrm{C}$. As amostras foram analisadas em tubo de $5 \mathrm{~mm}$ em 1,2,4-triclorobenzeno e benzeno deuterado, em quantidade equivalente a $30 \% \mathrm{em}$ volume.

As análises térmicas dos polímeros obtidos foram realizadas em um aparelho Dupont 2910, com taxa de aquecimento e resfriamento de $10{ }^{\circ} \mathrm{C} / \mathrm{min}$, na faixa de $40-240{ }^{\circ} \mathrm{C}$, calibrado com índio.

As viscosidades intrínsecas $[\eta]$ dos polímeros foram determinados com o auxílio de um viscosímetro do tipo Ubbelohd, empregando-se o Decahidronaftaleno ou decalina como solvente a $135{ }^{\circ} \mathrm{C}$. O peso molecular viscosimétrico médio $\left(\mathrm{M}_{\mathrm{v}}\right)$ foi obtido através substituição dos parâmetros sugeridos por Kinsinger e Hughes ${ }^{31}$ na equação de Mark-Houwink, a saber: $[\eta]_{\left(\operatorname{Dec}, 135^{\circ} \mathrm{C}\right)}=1,10 \times 10^{-4}\left(\mathrm{M}_{\mathrm{v}}\right)^{0,80}$

\section{Resultados e Discussão}

A química para a síntese de complexos catalíticos metalocênicos é bastante sensível às condições experimentais, principalmente em se tratando dos cuidados necessários durante as reações de obtenção dos complexos e, até mesmo, dos intermediários. Os complexos catalíticos mais difíceis de serem sintetizados são aqueles que apresentam ponte interanelar e/ou quando os anéis ciclopentadienílicos são diferentes. Estas dificuldades se devem, principalmente, a reações secundárias que podem ocorrer nestes casos. Por exemplo, os anéis ciclopentadienílicos, de um mesmo ligante, podem estar ligados a dois centros metálicos e não a um único como é requerido. Os precursores catalíticos utilizados neste estudo, na polimerização de propeno, foram aqueles apresentados na Figura 1. 


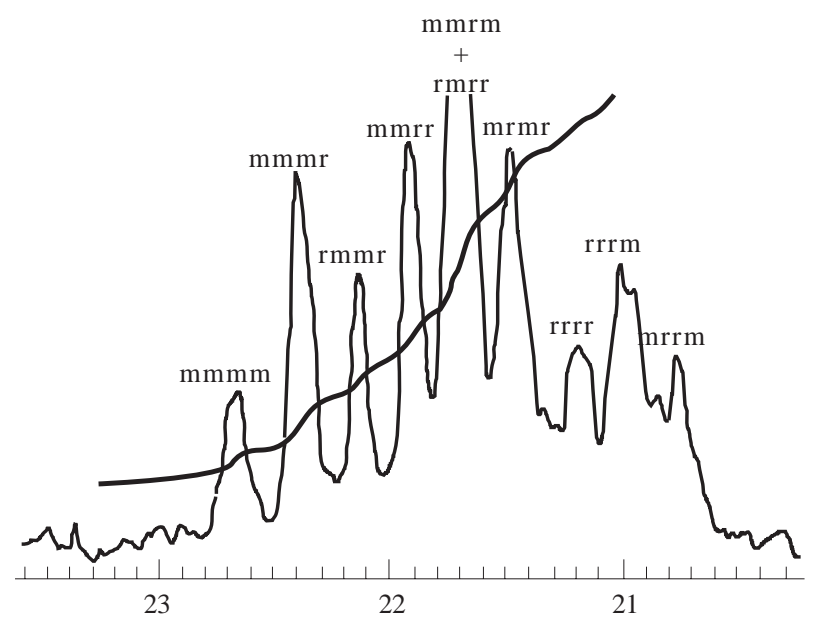

Figura 2. Espectro de $\mathrm{RMN}$ de ${ }^{13} \mathrm{C}$ de polipropileno atático ( $\operatorname{Ind}_{2} \mathrm{ZrCl}_{2}$ a $20{ }^{\circ} \mathrm{C}$ ).

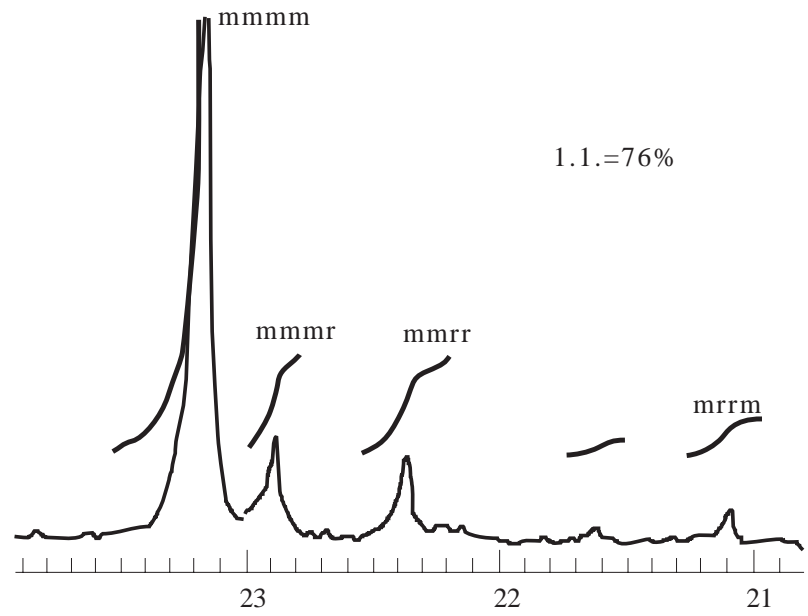

Figura 3. Espectro de $\mathrm{RMN}$ de ${ }^{13} \mathrm{C}$ de polipropileno isotático (Et(Ind) $\mathrm{ZrCl}_{2}$ a $50{ }^{\circ} \mathrm{C}$ ).

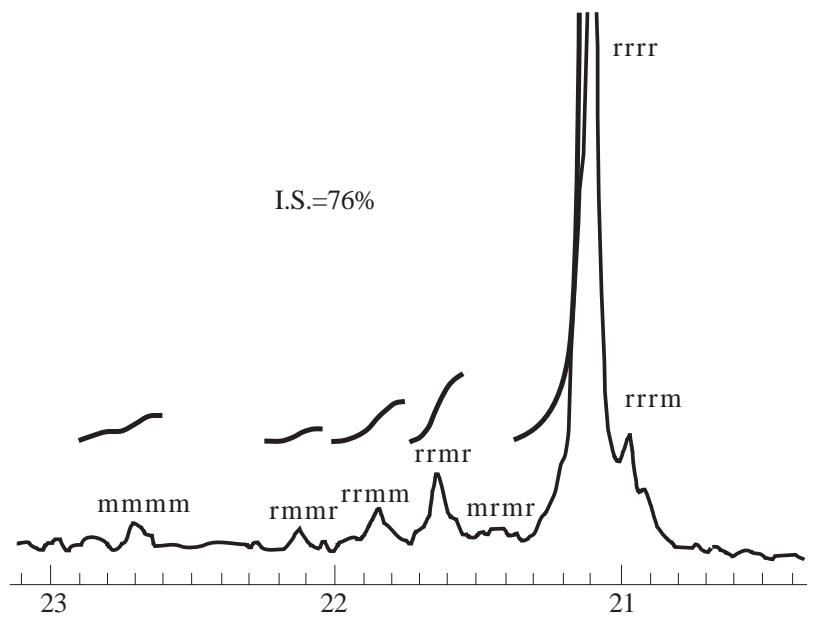

Figura 4. Espectro de $\mathrm{RMN}$ de ${ }^{13} \mathrm{C}$ de polipropileno sindiotático (iPrCpFluZrCl a $30{ }^{\circ} \mathrm{C}$ ).

Estudos preliminares com os catalisadores sintetizados, demonstraram que a temperatura de polimerização, entre outros fatores avaliados, era a única variável que causava maiores alterações nas características do polímero produzido. Estas alterações estão provavelmente relacionadas com a configuração/conformação do precursor metalocênico, 0 qual sofre modificação na sua estrutura com a temperatura, ocorrendo variação na sua fluxionalidade e consequentemente na estrutura do polímero formado, conforme salientado na introdução.

O cálculo percentual das áreas de cada pico do espectro de $\mathrm{RMN}$ de ${ }^{13} \mathrm{C}$ da região dos carbonos tipo $\mathrm{CH}_{3}$ para o polipropileno pode fornecer o grau de taticidade relativo a cada tipo de configuração presente no polímero. A configuração isotática é dada pelas pentades $\mathrm{mmmm}$, a sindiotática pelas pentades $r r r r$, e as demais por diferentes combinações de seqüências $m$ (meso) e $r$ (racêmica).

A Figura 2 mostra um espectro de $\mathrm{RMN}$ de ${ }^{13} \mathrm{C}$ típico de polipropileno atático produzido com o precursor catalítico 3, sem ponte, cocatalisados por MAO. Em todas as temperaturas testadas a atividade desses sistemas foi inexpressiva e somente oligômeros de polipropileno foram produzidos. A não formação de polímeros isotáticos com a diminuição da temperatura de polimerização, demonstra que esse precursor catalítico bem como o de número 4 não apresentam, em nenhum momento, conformação estereosseletiva com relação ao monômero incorporado, em temperaturas de até $5^{\circ} \mathrm{C}$.

As Figuras 3 e 4 mostram, respectivamente, espectros de $\mathrm{RMN}$ de ${ }^{13} \mathrm{C}$ de polipropileno isotático, obtido com o precursor catalítico $1 / \mathrm{MAO}$ a $50{ }^{\circ} \mathrm{C}$, e de polipropileno sindiotático obtido com o precursor catalítico $2 / \mathrm{MAO}$ a $30{ }^{\circ} \mathrm{C}$.

A variação do grau de taticidade dos polímeros produzidos com os sistemas isoespecífico 1/MAO e sindioespecífico $2 / \mathbf{M A O}$, com ponte, em função da temperatura de polimerização pode ser verificado na Tabela 1 , conforme já publicado anteriormente ${ }^{32}$. Tanto a atividade do catalisador como o peso molecular do polímero foram influenciados pela temperatura de polimerização.

A diminuição da estereorregularidade do polímero, com o aumento da temperatura, pode ser explicada como conseqüência do aumento da fluxionalidade da estrutura do catalisador e da energia do sistema, permitindo dessa forma, coordenações e inserções dos monômeros que, a mais baixas temperaturas, dificilmente ocorreriam. Com a diminuição da estereoespecificidade do catalisador, 
Tabela 1. Polimerização de propeno com os sistemas isoespecífico $\mathrm{Et}(\mathrm{Ind})_{2} \mathrm{ZrCl}_{2} / \mathrm{MAO}$ (1) e sindioespecífico iPrCpFluZrCl${ }_{2} / \mathrm{MAO}$ (2).

\begin{tabular}{|c|c|c|c|c|c|c|}
\hline \multirow[t]{2}{*}{$\mathrm{T}\left({ }^{\circ} \mathrm{C}\right)$} & \multicolumn{2}{|c|}{$\begin{array}{l}\text { Ativ. catalítica } \\
\text { (kgPP/molZr.h) }\end{array}$} & \multicolumn{2}{|c|}{$\begin{array}{l}\text { Peso molecular } \\
\text { viscosimétrico } \\
\text { médio (Mv) }\end{array}$} & \multirow{2}{*}{$\begin{array}{c}\begin{array}{c}\text { Índice de } \\
\text { isotaticidad- } \\
\text { e mmmm } \\
(\%)\end{array} \\
1\end{array}$} & \multirow{2}{*}{$\begin{array}{c}\text { Índice de } \\
\text { sindiotatic- } \\
\text { idade rrrr } \\
(\%) \\
2\end{array}$} \\
\hline & 1 & 2 & 1 & 2 & & \\
\hline 10 & 33 & 340 & & $\begin{array}{c}1126- \\
00\end{array}$ & & 89 \\
\hline 20 & 930 & 571 & $\begin{array}{l}2730- \\
0\end{array}$ & 93300 & 88 & 84 \\
\hline 30 & 3870 & 1000 & $\begin{array}{c}1900- \\
0\end{array}$ & 41100 & & 76 \\
\hline 40 & 7200 & 720 & $\begin{array}{c}1500- \\
0\end{array}$ & 42000 & 79 & \\
\hline 50 & 5950 & 290 & $\begin{array}{c}1050- \\
0\end{array}$ & 14100 & 76 & 65 \\
\hline 60 & 5630 & & 5600 & & 74 & \\
\hline
\end{tabular}

Condições de polimerização de propeno (reator de 0,5L): Sistema isoespecífico: $\mathrm{Tol}=250 \mathrm{~mL} ;[\mathrm{Zr}]=33.10^{-6} \mathrm{~mol} / \mathrm{L} ; \mathrm{Al} / \mathrm{Zr}=1440$; tempo $=120 \mathrm{~min}$. Sistema sindioespecífico: $\mathrm{Tol}=150 \mathrm{~mL} ; \mathrm{Zr}]=66.10^{-6} \mathrm{~mol} /$ $\mathrm{L} ; \mathrm{Al} / \mathrm{Zr}=750$; tempo de $120 \mathrm{~min}$.

ocorre um aumento de produção de cadeias com diferentes microestruturas, acarretando uma diminuição do percentual relativo de pentades $\mathrm{mmmm}$ ou $r r r$, referentes respectivamente as configurações isotáticas e sindiotáticas.

A variação do peso molecular para o sistema 2/MAO, apresentado na Tabela 1, são as mesmas que para o sistema 1/MAO. Os valores de peso molecular (Mv) dos polímeros obtidos com o sistema catalítico sindioespecífico 2/MAO foram sempre superiores aos obtidos com o sistema isoespecífico 1/MAO, em todas as temperatura avaliadas. Esta variação esta relacionada com o aumento da velocidade das reações de terminação de cadeia do tipo $\beta$-eliminação, com a formação de hidretos metálicos e liberação da cadeia em crescimento. A formação de hidretos metálicos pode contribuir também, para o aumento da atividade de polimerização, uma vez que estes são espécies ativas extremamente reativas. A temperaturas muito altas esses sistemas apresentam uma menor atividade provavelmente devido à desativação ou decomposição das espécies ativas e também pela diminuição da concentração do monômero dissolvido no meio, uma vez que o sistema não é pressurizado.

Os catalisadores testados não permitem um controle do peso molecular dos polímeros pela adição de um agente de transferência de cadeia, por exemplo hidrogênio, como praticado industrialmente com os catalisadores Ziegler-Natta, devido ao fato destes
Tabela 2. Variação da temperatura de fusão $\left(T_{m}\right)$ e cristalização $\left(T_{c}\right)$ dos polipropilenos obtidos em função da temperatura de polimerização (Tp). Sistema catalítico Et(Ind) ${ }_{2} \mathrm{ZrCl}_{2} / \mathrm{MAO}$.

\begin{tabular}{|c|c|c|c|c|}
\hline CAT. & $\operatorname{Tp}\left({ }^{\circ} \mathrm{C}\right)$ & $\operatorname{Tm}_{1}\left({ }^{\circ} \mathrm{C}\right)$ & $\mathrm{Tm}_{2}\left({ }^{\circ} \mathrm{C}\right)$ & $\operatorname{Tc}\left({ }^{\circ} \mathrm{C}\right)$ \\
\hline \multirow{4}{*}{$\mathrm{Et}(\mathrm{Ind})_{2} \mathrm{ZrCl}_{2}$} & 10 & 142,9 & 140,5 & 107,2 \\
\hline & 20 & 138,9 & 139,1 & 105,7 \\
\hline & 40 & 127,5 & 128,7 & 95,9 \\
\hline & 50 & 122,1 & 119,6 & 87,8 \\
\hline
\end{tabular}

serem muito baixos. Para se ter um controle do peso molecular do polímero pela adição de um agente de transferência de cadeia, é necessário que o catalisador na ausência do mesmo produza polímero com alto peso molecular ou baixo índice de fluidez.

A Tabela 2 mostra os valores de temperatura de fusão e cristalização, determinados por DSC dos polímeros preparados com o sistema isoespecífico a diferentes temperaturas. Considerando-se que todas as condições de reação foram idênticas é possível observar-se para o sistema isoespecífico, que a medida que a temperatura de polimerização aumenta a $\mathrm{T}_{\mathrm{m}}$ e a $\mathrm{T}_{\mathrm{c}}$ dos polímeros obtidos diminuem. Essa variação está de acordo com a do peso molecular e da estereorregularidade do polímero com o aumento da temperatura de polimerização.

Como mostram os resultados acima apresentados aumentos de $10^{\circ} \mathrm{C}$ na temperatura de polimerização, acarretaram diferenças significativas nas características dos polímeros obtidos, ocorrendo uma diminuição da estereorregularidade, peso molecular e temperaturas de fusão $\left(\mathrm{T}_{\mathrm{m}}\right)$ e cristalização $\left(\mathrm{T}_{\mathrm{c}}\right)$ dos polipropilenos produzidos com os catalisadores de metalocenos testados.

\section{Conclusões}

O peso molecular viscosimétrico médio $\left(\mathrm{M}_{\mathrm{v}}\right)$ dos polímeros obtidos com os sistemas estereoespecíficos apresentaram valores de $M_{v}$ na faixa de 5.600 a $112.600 \mathrm{~g} / \mathrm{mol}$ e temperatura de fusão $\left(\mathrm{T}_{\mathrm{m}}\right)$ e $115 \mathrm{a}$ $143{ }^{\circ} \mathrm{C}$. Ambos são considerados baixos se comparados aos obtidos com catalisadores ZieglerNatta heterogêneos que ficam na faixa de 100.000 a $1.000 .000 \mathrm{~g} / \mathrm{mol} \mathrm{e} 163{ }^{\circ} \mathrm{C}$, respectivamente. 
O teor de polímero estereoespecífico no produto final variou com a variação da temperatura de polimerização da alfa-olefina.

O sistema metalocênico sindioespecífico mostrase extremamente promissor para a obtenção de polímeros sindiotáticos a base de $\alpha$-olefinas, se comparado com o sistema sindioespecífico a base de vanádio $\mathrm{VCl}_{4} / \mathrm{AlEt}_{2} \mathrm{Cl}$. Este último, além de apresentar baixíssima atividade, apresenta condições extremas de polimerização, necessitando temperaturas muito baixas da ordem de $-70{ }^{\circ} \mathrm{C}$ para produzir polímero estereorregular.

\section{Referências Bibliográficas}

1. Kely, T.J.; Pauson, P.J.; -Nature (London), 168, 1039,(1959);

2. Miller, S.A.; Tebboth, J.A.; TREMAINE, J.F.; J. Chem. Soc., 632, (1952);

3. Möhring, P.C.; Coville, N.J.; -J. Organomet. Chem., 479, 1, (1994);

4. Corradini, P.; Guerra, G.; Villani, V.; Vacatello, M.; -Gazz. Chim. Ital., 118, 173,(1988);

5. Corradini, P.; Guerra, G.; Cavallo, L.; Vacatello, M.; -Polymer, 32, 1329, (1991);

6. Cosse, P.; -"The Stereochemistry of Macromolecules" (A.D.Ketley Ed.), Vol. 1, Chap. 3, Marcel Dekker, New York,(1967);

7. Hine, J.; -Adv. Phys. Org. Chem., 15, 1, (1977);

8. Corradini, P.; Guerra, G.; Cavallo, L.; Vacatello, M.; -Macromolecules, 24, 1784, (1991);

9. Lee, I.; Gauthler, W.J.; Ball, J.M.; Iyengar, B.; Collins, S.; -Organometallics, 11, 2115 (1992);

10. Ravazi, A.; -J.Am.Chem.Soc., 115, 7529, (1993);

11. Ewen, J.A.; Jones, R.L.; Razavi, A.; Ferrara, J.D.; -J. Am. Chem. Soc., 110, 6255, (1988);

12. Razavi, A.; E.P. 0426643 A1, (1991);

13. Ewen, J.A.; -J. Am. Chem. Soc., 106, 6355 (1984);

14. Ewen, J.A.; Elder, M.J.; Jones, R.L.; Curtis, S.; Cheng, H.N.; -"Catalytic Olefins Polymerization", Ed. T.Keii and K.Soga, 56, 439 (1990);
15. Barbé, P.C.; Cecchin, G.; Noristi, L.; -Adv. Polym. Sci., 81, 1 (1987);

16. Tsutsui, T.; Mizuno, A.; Kashiwa, N.; -Polymer, 30, 428 (1989);

17. Grassi, A.; Zambelli, A.; Resconi, L.; Albizzati, E.; Mazzocchi, R.;-Macromolecules, 21, 617 (1988);

18. Perrin, D.D.; Armarengo, W.L.F.; -"Purification of Laboratory Chemicals", 3rd. ed., Pergamon Press, 1988, N.Y.;

19. Ewen, J.A.; Elder, M.J.; Jones, R.L.; Curtis, S.; Cheng, H.N.; -"Catalytic Olefins Polymerization", Ed. T.Keii and K.Soga, 56, 501, (1990);

20. Collins, S.; Kuntz, B.A.; Taylor, N.J.; Ward, D.G.; -J. Organomet. Chem., 342, 21, (1988);

21. Grossman, R.B.; Doyle, R.A.; Buchwald, S.L.; Organometallics, 10, 1501, (1991);

22. Wild, F.R.W.P.; Zsolnai, L.; Huttner, G.; Brintzinger, H.H.; -J. Organomet. Chem., 232, 233, (1982);

23. Wild, F.R.W.P.; Wasiucionek, M.; Huttner, G.; Brintzinger, H.H.; -J. Organomet. Chem., 288, 63, (1985);

24. Wilkinson, G.; Birmingham, J.M.; -J. Am. Chem. Soc. 76, 4281, (1954);

25. Piccolrovazzi, N.; Pino, P.; Consiglio, G.; Sironi, A.; Moret, M.; -Organometallics, 9, 3098, (1990);

26. Samuel, E,; Rausch, M.D.; -J. Am. Chem. Soc., 95, 6263, (1973);

27. Khera, B.; Kaushik, N.K.; -Bull. Soc. Chim. Fr., 11-12, 278, (1983);

28. Samuel, E.; Setton, R.; -J. Organomet. Chem., 4, 156, (1965);

29. Chien, J.C.W.; Rieger, B.; Sugimoto, R.; Mallin, D.T.; Rausch, M.D.; -Catalytic Olefin Polimerization, Ed.T.Keii, K.Soga, 56, 535 (1990);

30. Manzer, L.E.; -Inorg. Synth., 21, 135 (1982);

31. Kinsiger, J.B.; Hughes, R.E.; -J. Phys. Chem. 63, 2002 (1959);

32. Meneghetti, M.R.; Forte, M.C.; Dupont, J.; Polym. Bulletin, 35, 431, (1995). 